\title{
Process Optimization and Properties of Magnetically Hard Cobalt Carbide Nanoparticles via
}

\section{Modified Polyol Method}

Mehdi Zamanpour, ${ }^{\mathrm{a}, *}$ Steven P. Bennett, ${ }^{\mathrm{a}}$ Leily Majidi, ${ }^{\mathrm{b}}$ Yajie Chen ${ }^{\mathrm{a}}$ and Vincent G. Harris ${ }^{\mathrm{a}, \mathrm{c}}$

${ }^{a}$ Center for Microwave Magnetic Materials and Integrated Circuits (CM3IC), Northeastern University, Boston, MA 02115, USA

${ }^{\mathrm{b}}$ Department of Mechanical and Industrial Engineering, Northeastern University, Boston, MA 02115, USA

${ }^{\mathrm{c}}$ Department of Electrical and Computer Engineering, Northeastern University, Boston, MA 02115, USA

ABSTRACT: Cobalt carbide magnetic nanoparticles were successfully synthesized via a modified polyol process without using a rare-earth catalyst during the synthesis process. The present results show admixtures of $\mathrm{Co}_{2} \mathrm{C}$ and $\mathrm{Co}_{3} \mathrm{C}$ phases possessing magnetization values exceeding $47 \mathrm{emu} / \mathrm{g}$ and coercivity values exceeding $2.3 \mathrm{kOe}$ at room temperature. Moreover, these experiments have illuminated the important role of the reaction temperature, hydroxyl ion concentrations and the reaction duration on the crystallographic structure and magnetic properties of the nanoparticles. The crystallographic structure and particle size of the $\mathrm{Co}_{\mathrm{x}} \mathrm{C}$ nanoparticles were characterized by $\mathrm{X}$ ray diffractometry and scanning electron microscopy. Vibrating sample magnetometry was used to determine magnetic properties. Scale-up of synthesis to more than 5 grams per batch was demonstrated with no significant degradation of magnetic properties.

\section{Introduction}

There has been a large amount of research devoted to seeking alternates to rare earth magnets since these are expensive, prone to corrosion and, experience as such, severe cost limitations as well as supply chain challenges. Although much attention has been paid to carbon-containing magnetic materials which include carbon-coated magnetic-metal nanocrystalline materials [1], $\mathrm{M}_{\mathrm{n}} \mathrm{C}(\mathrm{M}=\mathrm{Fe}, \mathrm{Co}$, $\mathrm{Ni}, \mathrm{Cu}, \mathrm{n}=1-6)$ nanoclusters [2] and $\mathrm{Co}-\mathrm{C}$ granular films [3-5], these studies did not explore their magnetic properties. The focus of those studies was the fabrication of carbon-related composites mostly for biomedical applications and cancer remediation therapies [6].

Historically, cobalt carbide was studied because of its ready formation during Fischer-Tropsch (FT) synthesis of methane [7] which can be described as a polymerization reaction of adding $\mathrm{C}_{1}$ (simple alkyl chain) unit to a growing chain. In that and related cases, the surface of the 
catalyst always consists of a wide range of carbon-containing molecules with each interacting with the catalyst differently. Therefore, it is reasonable to expect that carbon is a likely cause of deactivation of the catalysts by the formation of carbides [8-12]. X-ray diffraction (XRD) was utilized as the most commonly used technique to detect carbides [13 \& 14]; however, cobalt carbides were found to be metastable especially in the presence of $\mathrm{H}_{2}$ [15]. It was also found that cobalt crystallites subjected to pure carbon monoxide at $226-230{ }^{0} \mathrm{C}$ slowly form $\mathrm{Co}_{2} \mathrm{C}$. Fukumiya et al. [16] studied the thermal stability of metastable co-sputtered Co-C composite alloy films in terms of the concept of formation enthalpy. They also reported the mechanical hardness of the alloy films and found it to decrease near linearly with increasing carbon content in the film, concluding that this carbon content can be used as the measurement of hardness to monitor film qualities. Furthermore, they showed that the hardness of metastable phase is greater than the alloy films. Another result [17] showed formation of Co- $\mathrm{C}_{60}$ polymeric chains as the major product of co- deposition of Co and fullerene instead of metastable carbide phases. Upon annealing at 300 ${ }^{0} \mathrm{C}$, the growth of diamond and single-wall carbon nanotubes was observed. Wang et al. [18] reported fabrication of carbon-coated cobalt nanocapsules by the chemical vapor condensation process. The core-shell phases (onion-like morphology of alternating layers) were composed of fcc-Co, hcp-Co and cobalt carbides regardless of the condensation temperature. There are a few published articles on electronic structure of cobalt carbides [19-22]. Among them, Yong-Hui et al. [23] studied the structural and electronic properties of $\mathrm{Co}_{2} \mathrm{C}$ and its surface stability by density functional theory calculations. They found that the formation of $\mathrm{CO}_{2} \mathrm{C}$ phase is exothermic with the formation energy of 0.81 $\mathrm{eV} / \mathrm{Co}_{2} \mathrm{C}$ with respect to $\mathrm{Co}$ under the presence of $\mathrm{CO}$ and $\mathrm{H}_{2}$ gas stream. But they concluded that the cobalt carbide is a paramagnetic phase. Chemical techniques were not the only route to synthesize cobalt carbides. There have been some attempts to fabricate $\mathrm{Co}_{\mathrm{x}} \mathrm{C}$ materials by mechanical alloying as well [24 \& 25]. Nonequilibrium, f.c.c. CoC solid solutions 
supersaturated with carbon to above 7 at. \% was prepared by ball milling. By increasing the carbon content to 10 at. \% , the f.c.c. phase began converting to metastable $\mathrm{Co}_{3} \mathrm{C}$ with an orthorhombic structure. Further increasing the carbon content, milling time and heat treating the powders at different temperatures, fabrication of $\mathrm{Co}_{2} \mathrm{C}$ and $\mathrm{Co}_{3} \mathrm{C}$ was observed. Diaz BarrigaArceo et al. used the same technique to prepare cobalt carbide, although their objective was synthesis of carbon nano-fiber by decomposition of carbide phase by heating at 800 and $1000{ }^{\circ} \mathrm{C}$. However, no magnetic studies were performed.

Early studies by Chinnasamy et al. [26] reported a ferromagnetic material based upon nanoscale cobalt carbide particles that showed promise as a rare earth-free alternative to high performance permanent magnets. They fabricated cobalt carbide nanoparticles using polyol reduction chemistry in a single step process. Particles were acicular in morphology and room temperature coercivities reached $3.4 \mathrm{kOe}$ and maximum energy products greater than $20 \mathrm{kJm}^{-3}$ which is comparable with AlNiCo and ferrites. The particles of previous studies [ $\left[\begin{array}{lll}27 & \& & 28\end{array}\right]$ consisted of an admixture of $\mathrm{Co}_{2} \mathrm{C}$ and $\mathrm{Co}_{3} \mathrm{C}$ phases formed in the presence of Sm as catalyst. In this paper, we report the optimized fabrication of magnetically hard cobalt carbide nanoparticles by modified polyol method without using rare earth elements during the synthesis processes.

\section{Experimental Section}

2.1 Materials: Cobalt acetate tetrahydrate (Acros, 98\%), Tetraethylene Glycol (Acros, 99.5\%), Polyvinylpirrolidone (PVP, Mw=55000, Sigma Aldrich), Sodium Hydroxide (Pellets, Fisher), Potassium Hydroxide (Crystals, 99\% Fisher), Oleylamine (90\%, Acros), Reagent alcohol (Histological grade, 90\% Anhydrous ethyl alcohol, 5\% Methyl alcohol, 5\% Isopropyl alcohol, Fisher) were used as supplied without further purification.

Synthesis of cobalt carbide: nanoparticles were synthesized using a modified, one-pot polyol technique [29 \& 30]. In most of the experiments, unless stated otherwise, different amounts of cobalt acetate tetrahydrate, sodium hydroxide and polyvinylpyrrolidone (PVP) were added to 150 $\mathrm{mL}$ tetraethylene glycol (TEG) at room temperature, which served as solvent and 
reducing agent. The mixture was then stirred mechanically for $20 \mathrm{~min}$ to become homogenized. The solution was then heated to $120{ }^{0} \mathrm{C}$ to evaporate the water content in metal salt and the solvent. After $20 \mathrm{~min}$, the mixture was heated to the reaction temperature (290-310 ${ }^{0} \mathrm{C}$ ) and allowed to react for different reaction times. The solution was then cooled to room temperature by removing the heat source and a black solid precipitate was extracted by using a rare earth permanent magnet. After removing the supernatant, the concentrated solution was rinsed by reagent grade alcohol and centrifuged numerous times. Drying the black solid in air overnight was the last step to obtain the powders.

\subsection{Structural Characterization: X-ray} diffractometer XRD, (Rigaku, Ultima III, CuK $\alpha$ radiation) was used for phase identification, and determined the crystallinity of particles and purity of the samples. A copper anode was used as the source of x-rays with a current of $44 \mathrm{~mA}$ at an accelerating voltage of $40 \mathrm{kV}$ with the copper source emitting $\mathrm{x}$-rays with $\mathrm{K}_{\alpha 1}$ and $\mathrm{K}_{\alpha 2}$ wavelengths of $1.5405 \AA$ and $1.544 \AA$, respectively. A continuous scan mode was employed, in which the $\mathrm{x}$-ray source and the $\mathrm{x}$ ray detector were continuously scanned in $2 \theta$ utilizing a Bragg-Brentano scattering geometry. The dried powder samples were gently ground using a mortar and pestle and then pressed onto a low background, low volume holder using a Fisherbrand pre-cleaned microscope slide. The sample holder was then placed in the instrument for XRD analysis. Upon completion of the run, the data were analyzed using the Jade program, which has a built-in diffraction library to compare the sample's diffraction pattern to the diffraction patterns in the JCPDS-ICDD database.

\subsection{Magnetic Characterization: Room} temperature vibrating sample magnetometry (VSM) and physical property measurement system (PPMS) magnetometer were used to determine the magnetic properties of materials. A Lakeshore model 7300 VSM and a Quantum Design model 6000 PPMS were used for magnetic measurement at high fields.

\section{Results and Discussions}

It has been shown that cobalt-carbides consist of two exchange-coupled phases [27], $\mathrm{Co}_{2} \mathrm{C}$ and $\mathrm{Co}_{3} \mathrm{C}$ in which $\mathrm{Co}_{2} \mathrm{C}$ has lower magnetic moment 
but higher coercivity. The hypothesized reaction mechanism [31] of formation of $\mathrm{Co}_{\mathrm{x}} \mathrm{C}$ in tetraethylene glycol, TEG, consists of 4 steps: 1dissociation of cobalt salt to form cobalt ions, 2nucleation and growth of Co particles, 3decomposition of TEG on cobalt surface and 4carbon diffusion into cobalt lattice. It was predicted that $\alpha$-cobalt results in formation of $\mathrm{Co}_{3} \mathrm{C}$ and $\beta$-cobalt yields $\mathrm{Co}_{2} \mathrm{C}$ and $\mathrm{Co}_{3} \mathrm{C}$. By changing the reaction temperature, reaction duration, hydroxyl ion and carbon concentration and surfactant concentration, reaction mechanism, structure and magnetic properties and chemical composition of the resulted cobaltcarbides was studied.

\subsection{Reducing agent}

Reducing potential of the polyols strongly depends on the molecular weight/chain length. The longer the polyol chain, the greater efficacy the polyol has to reducing metal salts. The polyols studied in this work are the most widely used ones; ethylene glycol and tetraethylene glycol. In both cases, all operating conditions were the same. After measurement, the particle formed by ethylene glycol showed magnetically soft behavior. X-ray diffractograms show formation of metallic cobalt by ethylene glycol while tetraethylene glycol produced cobalt carbide nanoparticles. Table 1 compares these cases. It can be concluded that although both solvents were able to dissociate the metallic salt and precipitate cobalt, ethylene glycol did not decomposed and therefore was unable to provide carbon for formation of cobalt carbide, while the polyol with longer alkyl chain (more carbon) dissociated to synthesize the carbide species.

\subsection{Reaction time}

As discussed elsewhere [32], the rate of reduction of the particles, and therefore their shape, size and properties is a function of the reaction kinetics. One of the most important parameters is the reaction time. By implementing the reaction for various times, volume fraction of each carbide phase and formation of secondary can be controlled since the last step of the reaction; the extent carbon diffuses into cobalt lattice, can determine the fraction of each cobaltcarbide phase. On one hand, long reactions produce cobalt and carbides, while on the other hand short reactions inhibit complete diffusion of 
Table 1. Comparison of cobalt carbide particles synthesized by different polyols phases

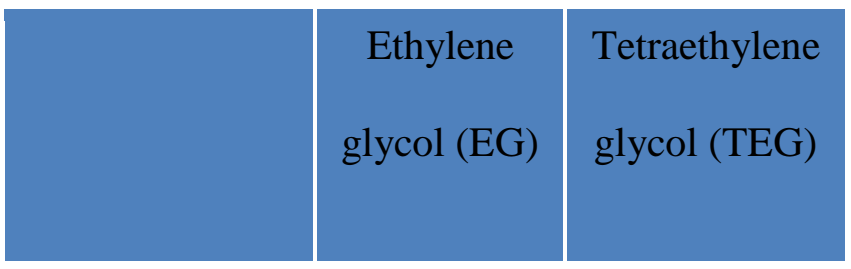

Reaction

Temperature $\left({ }^{0} \mathrm{C}\right)$

200

300

Saturation

Magnetization

104

46

(emu/g)

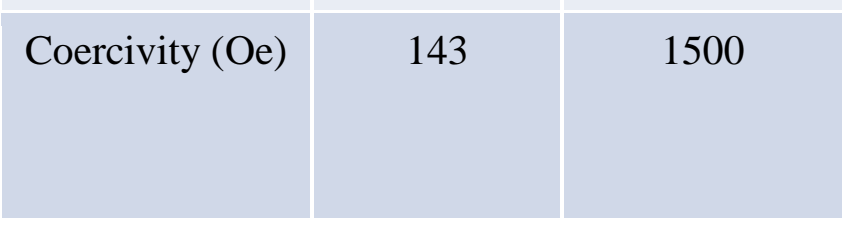

carbon into the cobalt lattice and results in cobalt-carbide-carbon core-shell nanostructures.

To study the effect of reaction time, raw materials were reacted for different periods of time between 5 minutes to 150 minutes. As seen in Figure 1, at short reaction times the magnetic moment and coercivity were low due to the formation of carbon-coated superparamagnetic cobalt particles. By increasing the reaction time, hence particles growth, the coercivity enhanced and reached its maximum at 1400 Oe at 45 minutes reaction, but decreased sharply and fell below 500 Oe after 75 minutes. Meanwhile, the magnetic moment started at more than 100 emu/g, which later confirmed by XRD to be dominated by metallic cobalt particles. According to the proposed reaction mechanism discussed earlier, after injecting the cobalt salt into the solution, it dissociates to cobalt ions which form nuclei. By increasing the concentration of these nuclei, cobalt particles form. Subsequently, by dissociation of the TEG into carbon, cobalt particles become coated by a layer of nonmagnetic carbon. The sharp drop in the moment of the samples to $30-40 \mathrm{emu} / \mathrm{g}$ is attributed to formation of these species that lasted until carbon began to diffuse into the cobalt lattice. Upon completion of this step, the coercivity reached its maximum while saturation magnetization changed insignificantly, but again increased abruptly to $90 \mathrm{emu} / \mathrm{g}$ at longer reaction times. X-ray diffraction scans show that by increasing the reaction time to over 90 minutes, some part of the cobalt-carbide decomposed to metallic cobalt and as a result, the moment 
increased and the coercivity decreased dramatically.

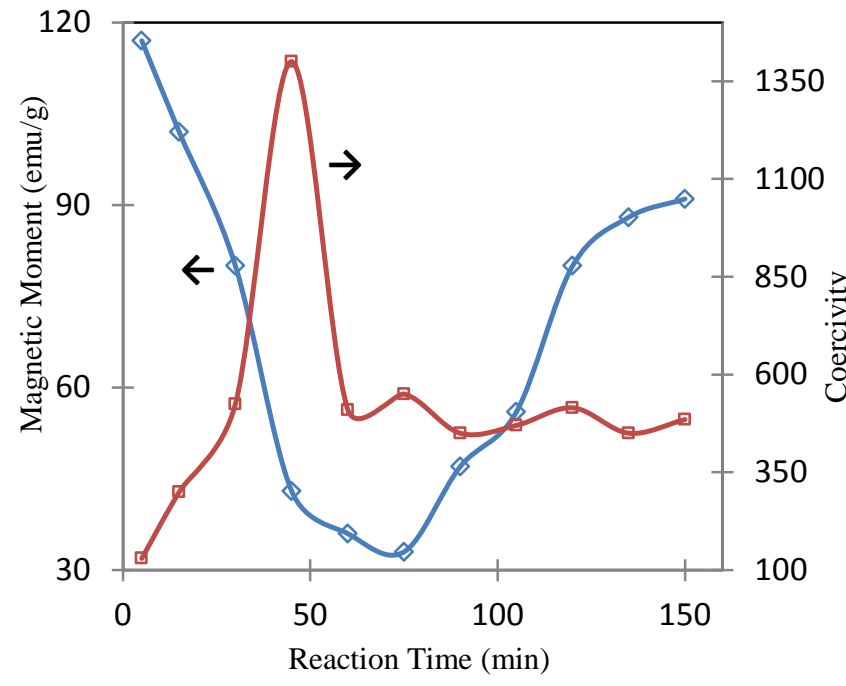

Figure 1. Change in saturation magnetization and coercivity of cobalt carbides with respect to reaction time. Blue curve shows the saturation magnetization and red curve indicates the coercivity of the cobalt carbide nanoparticles

\subsection{Reaction temperature}

After choosing the appropriate solvent and determining the ideal reaction time for synthesis of cobalt carbide, the reaction temperature was studied. It has been widely known that temperature plays a very important role in formation of metallic particles in solutions [33].

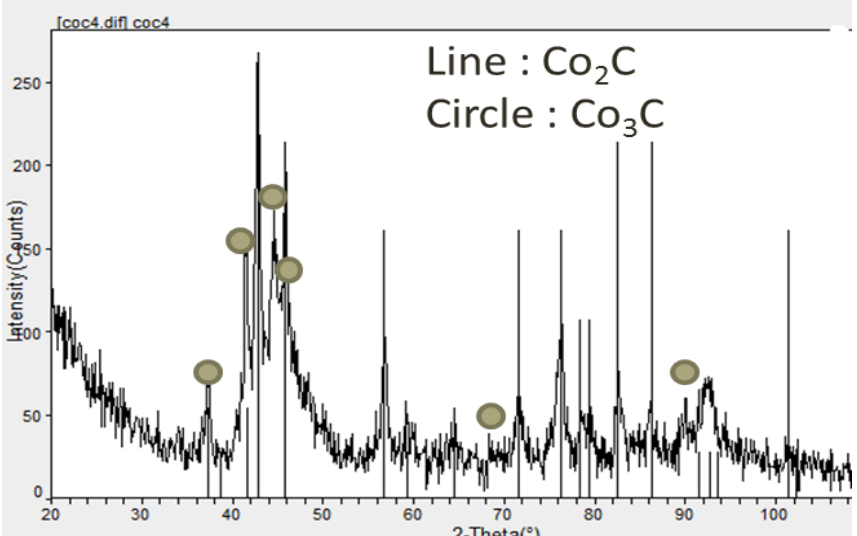

Figure 2. X-ray diffracogram for an admixture of $\mathrm{Co}_{2} \mathrm{C}$ and $\mathrm{Co}_{3} \mathrm{C}$ formed at 45 minutes reaction time. Note that no secondary phases detected other than cobalt carbide phases

The reaction temperature was changed between 240 and $310{ }^{0} \mathrm{C}$, which is the boiling point of the solvent, tetraethylene glycol. Magnetic and structural studies revealed that metastable cobalt carbide formation starts at $\sim 270{ }^{0} \mathrm{C}$ and at high end of the reaction temperature, the coercivity decreased from $1.6 \mathrm{kOe}$ to $1.2 \mathrm{kOe}$ which was attributed to the partial decomposition of cobalt carbides into cobalt-carbon core-shell structures which is in agreement with previous studies [34 \& 35]. 




Figure 3. Coercivity and saturation magnetization of cobalt carbide nanoparticles with respect to the reaction temperature. Blue curve shows magnetic moment and red line indicates coercivity of the samples

\subsection{Hydroxyl ion and carbon concentration}

$\mathrm{OH}$ ions provide the nucleation sites for formation of metallic nanoparticles in the solution. Therefore, hydroxyl concentration plays a crucial role in structure and compositional properties of the products. In order to investigate the effect of $\mathrm{OH}$ concentration of carbide formation, the $\mathrm{OH}$ : Co ratio was changed, keeping the Co content and the volume of the solution (C content) constant. To investigate the effect of carbon concentration, for the sake of accuracy, the same volume of solution was utilized. In each sets of experiments, while the amount of cobalt was changed, the $\mathrm{OH}$ : Co ratio was also adjusted to keep that ratio constant and therefore change the carbon content.

The initial cobalt content was $0.3 \mathrm{~g}$ and increased by $0.15 \mathrm{~g}$ increments until $0.75 \mathrm{~g}$. Figure 4 depicts the saturation magnetization of the nanoparticles versus hydroxyl ion concentration and cobalt content of the solution. The overall trend was to increase the saturation magnetization followed by a sharp decrease after reaching a maximum value. Although the magnetic moment fluctuated, it never recovered in magnitude. It should be noted that the maxima took place at different hydroxyl to cobalt ratios for different solutions but it usually shifted to larger ratios as the cobalt content increased, except for $0.75 \mathrm{~g}$ cobalt at which no absolute maximum was distinguished.

The trend in coercivity of the particles suggests more insight into the behavior of the system. In figure 5 one sees that the coercivity possesses an absolute maximum for each cobalt concentration. Figure 6 shows a hysteresis loop of sample 
containing 0.3 gram cobalt. Its shape and low coercivity indicated soft magnetic behavior of the particles. To shed more light, x-ray diffractometry was performed and no carbide peaks were detected. Based on these magnetic and structural results it can be concluded that at very low cobalt concentration, upon formation of small amount of cobalt nuclei, the subsequent particles grew rapidly which resulted in formation of larger particles. Carbon layers did not have enough time to diffuse into the cobalt lattice, resulting in the formation of carboncoated cobalt particles. Existence of this nonmagnetic layer was accounted for lower than expected saturation magnetization and the coercivity enhancement was not observed due to absence of hard phase cobalt carbide.

As more cobalt was added to the solution, the number of cobalt nuclei increased, subsequently this inhibited the formation of larger particles. This sluggish growth phase was in favor of coating the metallic particles by a carbon layer that was produced from the dissociation of the solvent, followed by diffusion of this layer into the cobalt lattice which led to formation of cobalt

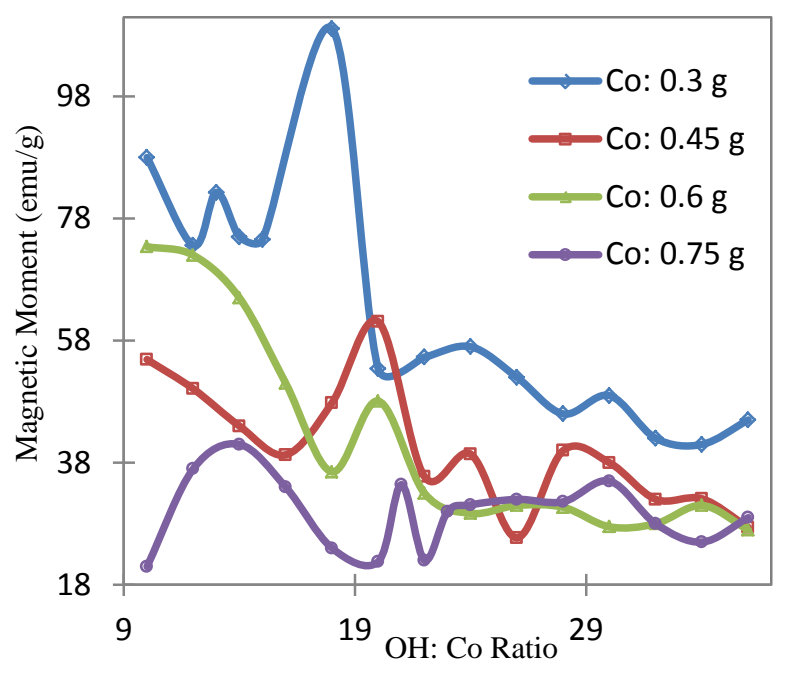

Figure 4. The effect of hydroxyl ions and carbon content on saturation magnetization of the particles for different amounts of cobalt



Figure 5. The effect of hydroxyl ions and carbon content on coercivity of the particles for different amounts of cobalt 
carbide nanoparticles. Synthesis of the cobalt carbide phases enhanced the coercivity at the expense of lowering the saturation magnetization.

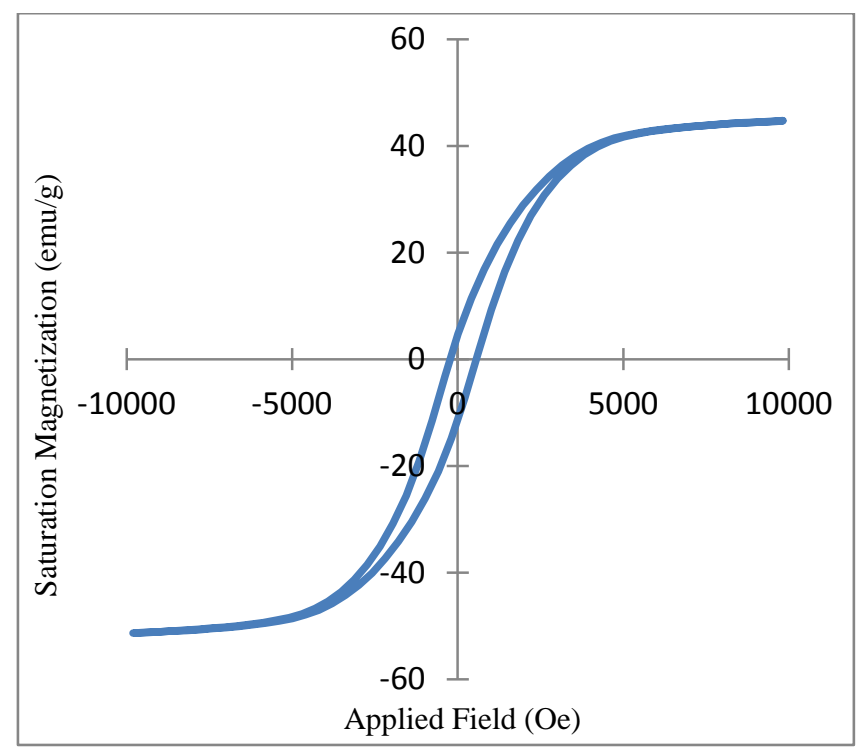

Figure 6. Hysteresis loop for a sample containing 0.3 grams cobalt as metal precursor and $\mathrm{OH}$ : $\mathrm{Co}$ ratio of 22:1. $\left(\mathrm{H}_{\mathrm{c}}=440\right.$ Oe and $\left.\mathrm{M}_{\mathrm{s}}=45 \mathrm{emu} / \mathrm{g}\right)$. Note the magnetically-soft behavior of the particles.

In each sets of experiments, as the hydroxyl ion to cobalt ratio increased, more nucleation sites became available for cobalt particles, paving the path to diffusion of carbon into smaller metallic particles and formation of carbide phase. Ample amounts of $\mathrm{OH}$ deteriorated the magnetic properties of the samples. This was mostly due to the large number of cobalt nuclei that made the solution carbon deficient. The carbon deficiency led to carbon-coated cobalt particles with soft magnetic properties. Overall, the optimum magnetic properties of the particles were realized at $\mathrm{OH}$ : Co range 20:1 to 26:1 in all experiments, but generally when more cobalt was present in the solution, that optimum ratio "window" was narrower. Figure 7 depicts a hysteresis loop for a hard-phase cobalt carbide sample with coercivity $2.3 \mathrm{kOe}$ and saturation magnetization $47 \mathrm{emu} / \mathrm{g}$. X-ray studies revealed the existence of equal volume fraction of $\mathrm{Co}_{2} \mathrm{C}$ and $\mathrm{Co}_{3} \mathrm{C}$ species in the sample. It should be noted that no secondary phases were observed in x-ray patterns. 


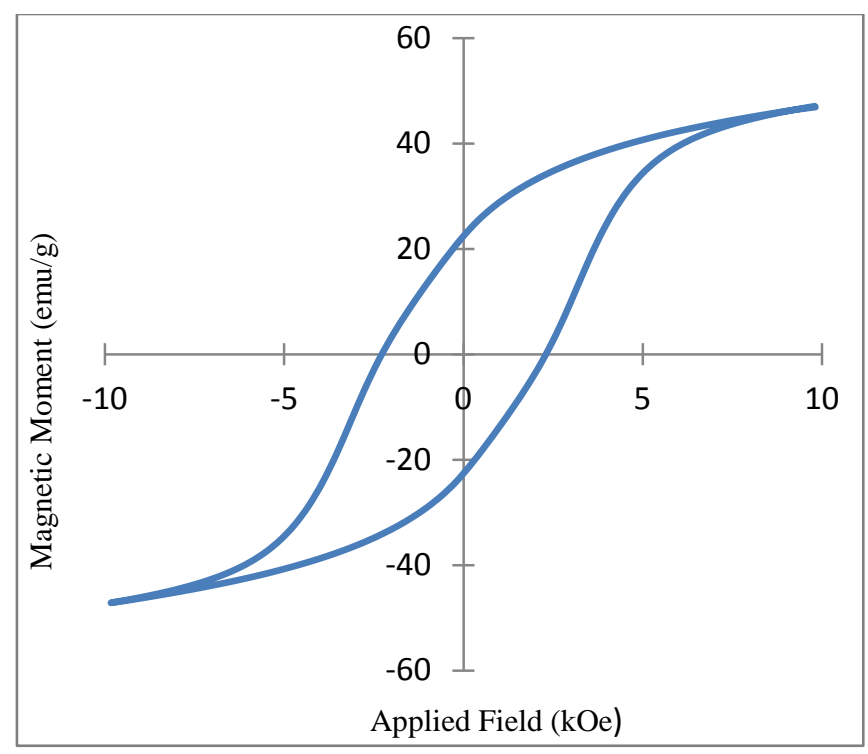

Figure 7. Hysteresis loop for a cobalt carbide sample with saturation magnetization $47 \mathrm{emu} / \mathrm{g}$ and coercivity $2.3 \mathrm{kOe}$

\subsection{Surfactant}

Two of the most common active agents or surfactants are PVP and Oleylamine (OA). These were chosen to investigate the effect of surfactant on the shape and size of the products hence the magnetic properties of the cobalt carbide nanoparticles. Although cobalt in its metallic form shows high affinity to oxidation, during the course of this work all the cobalt carbide samples were found oxide-free which indicated the ability of the solvents tetraethylene glycol, to serve both as reducing agent as well as capping agent/surfactant. To further control the size of the particles, PVP and Oleylamine (OA) were studied. In all experiments 0.6 grams cobalt were added to $150 \mathrm{~mL}$ TEG and reacted for 45 minutes. The results of magnetic properties of the particles are shown in Figure 8. While there was not detected any significant difference between the size of the particles (see Table 2), TEG proved to be the best surfactant because of very low magnetic moment of the particles synthesized in presence of PVP as surfactant. In case of OA, the coercivity of the particles was below the hard magnetic phase threshold. It's worth noting that both PVP and TEG showed similar patterns of change in $H_{c}$ and $M_{s}$, but particles formed in the presence of OA behaved differently, as seen in Figure 8. 

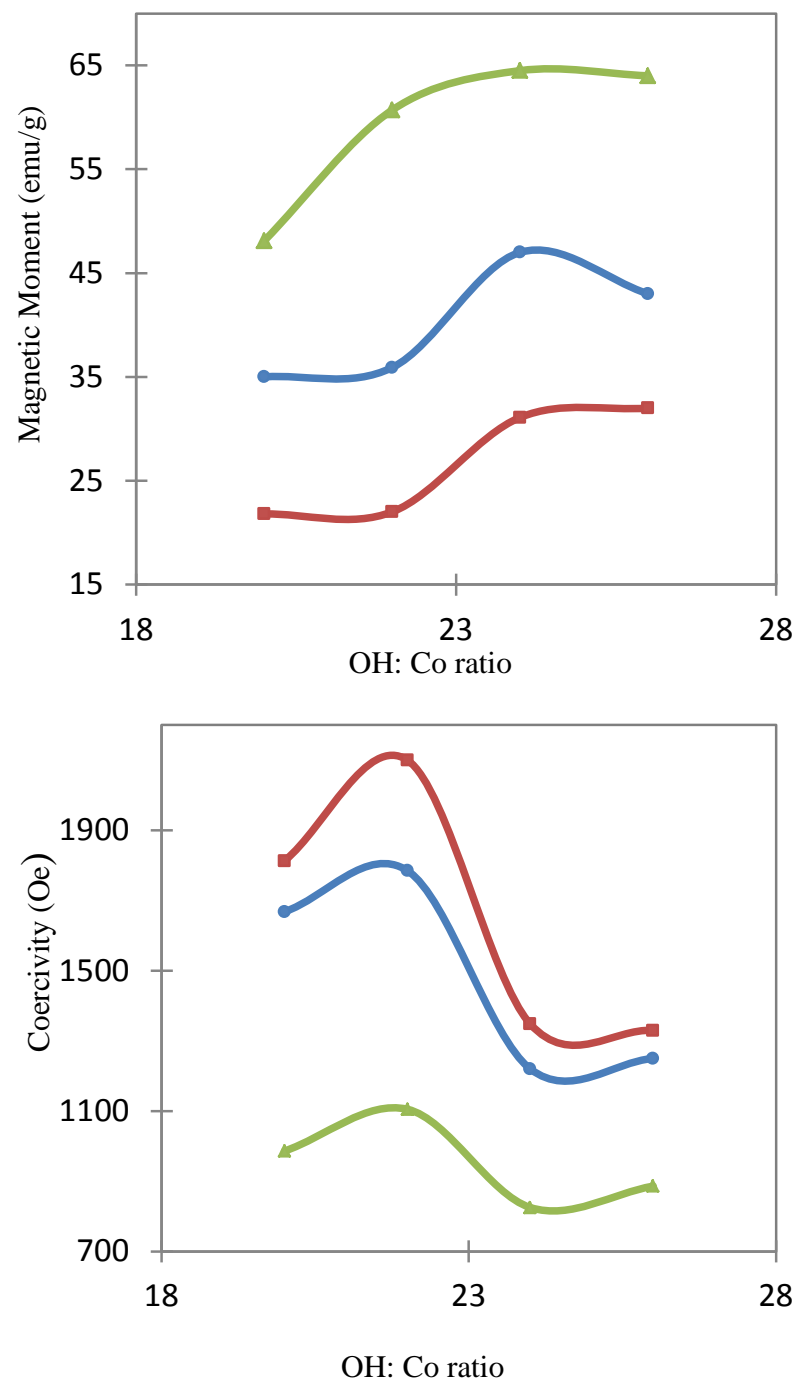

Figure 8. The effect of surfactant of (top) Saturation magnetization and (bottom) coercivity of the cobalt carbide particles. Oleyilamine, tetraethylene glycol and PVP are shown by triangles, circles and squares, respectively.
Table 2. Particle Size ( $D_{p}$ in nanometer) of cobalt carbide particles as a function of surfactant

OH: Co TEG PVP OA

\begin{tabular}{l|lll|}
20 & 33 & 32 & 37 \\
22 & 29 & 35 & 26 \\
24 & 22 & 33 & 33 \\
26 & 18 & 34 & 26 \\
\hline
\end{tabular}

\section{Conclusion}

Synthesizing a newly discovered hard magnetic cobalt carbide nanoparticles was achieved via modified polyol method using tetraethylene glycol (TEG) as solvent and reducing agent. Although previous studies suggested that rare earth metals are required to synthesize cobalt carbide and it was postulated that they act as catalyst in formation of the carbides, but in this study we have successfully fabricated cobalt carbide without need to utilize precious rare earth elements. Effect of different reaction kinetics parameters were studied and cobalt carbides particles with magnetic moment $47 \mathrm{emu} / \mathrm{g}$ and coercivity as high as $2.3 \mathrm{kOe}$ were 
fabricated. The temperature and reaction time found to be the most important parameters in determining properties of the final products. PVP and Oleylamine were used as surfactants and found to deteriorate magnetic properties of the particles, while TEG proved to serve as both reducing agent and surfactant with minimum adverse effect on coercivity and saturation magnetization.

\section{Acknowledgments}

We acknowledge funding from US Department of Energy’s Advanced Research Projects AgencyEnergy.

\section{References}

[1] Wang Z. H.; Choi C. J.; Kim B. K.; Kim J. C.; Zhang Z. D.; Characterization and magnetic properties of carbon-coated cobalt nanocapsules synthesized by the chemical vapor condensation process, Carbon, 2003; 41: 1751-1758

[2] Black S. J.; Morely C. P.; Owen A. E.; Elsegood M. R. J.; Synthesis and characterisation of phosphane-substituted $\mathrm{Co}_{3} \mathrm{C}$ clusters having a pendant allyl side-chain, Organometallic Chemistry, 2004; 689:2103-2113
[3] Konno T. J.; Shoji K.; Sumiyama K.; Suzuki K.; Structure and magnetic properties of cosputtered Co-C thin films, Magn. Magn. Matet., 1999; 195: 9-18

[4] Wang H.; Wong S. P.; Cheung W. Y.; Ke N.; Lau W. F.; Chiah M. F. et. al.; Structural and magnetic properties of Co65C35 nanocomposite films prepared by pulsed filtered vacuum arc deposition. Mater. Sci. Eng. C, 2001; 16: 147-151 [5] Zeng F. H.; Zhang X.; The magnetic properties and characterization of Co1- $\mathrm{x}-\mathrm{yCryCx}$ films produced by pulsed laser deposition. Magn. Magn. Mater., 2007; 309: 160168

[6] McHenry M. E.; Majetich S. A.; Artman J. O.; DeGraef M.; Staley S. W.; Superparamagnetism in carbon-coated Co particles produced by the Kratschmer carbon arc process. Phys. Rev. B, 1994; 49: 11358-11363

[7] Chaubey G. S.; Barcena C.; Paudyal N.; Rong C.; Gao J.; Sun S. et. al.; Synthesis and stabilization of FeCo nanoparticles Am. Chem. Soc., 2007; 129: 721-725 
[8] Menon P. G.; Coke on catalysts-Harmful, harmless, invisible and beneficial types. J. Mol.

Catal. A, 1990; 59: 207-209

[9] Moodley D. J.; Van de Loosdrecht J,;Saib A.

M.; Datye A. L.; Niemantsverdreit J. W.; On the

Deactivation of Cobalt-based Fischer-Tropsch Synthesis Catalysts. Am. Chem. Soc., Div. Petro.

Chem., 2008; 53: 122-125

[10] Lee D. K.; Lee J. H.; Ihm S. K.; Effect of Carbon Deposits on Carbon Monoxide Hydrogenation over Alumina-Supported Cobalt Catalyst. Appl. Catal., 1988; 36: 199-207

[11] Moodley D. J.; Van de Loosdrecht J.; Saib A. M.; Overett M. J.; Datye, A. L.; Niemantsverdreit J. W.; Carbon deposition as a deactivation mechanism of cobalt-based FischerTropsch synthesis catalysts under realistic conditions. Appl. Catal. A, 2009; 354: 102-110 [12] Barbier A.; Tuel A.; Arcon I.; Kodre A.; Martin G. A.; Characterization and Catalytic Behavior of $\mathrm{Co} / \mathrm{SiO}_{2}$ Catalysts: Influence of Dispersion in the Fischer-Tropsch Reaction. J. catal., 2001; 200: 106-116
[13] Hofer L. J. E.; Peebles W. C.; Preparation and X-Ray Diffraction Studies of a New Cobalt Carbide. J. Am. Chem. Soc., 1947; 69: 893-899 [14] Ducreux O.; Lynch J.; Rebours B.; Roy M.; Chaumette P.; In situ characterisation of cobalt based fischer-tropsch catalysts : A new approach to the active phase. Sur. Sci. Cat., 1998; 119: $125-130$

[15] Nagakura S.; Study of Metallic Carbides by Electron Diffraction Part IV. Cobalt Carbides. J. Phys. Soc. Jp., 1961; 16: 1213-1219

[16] Fukumiya Y.; Haga Y.; Nittono O.; Thermal stability of metastable Co-C composite alloy films. J. Mater. Sci. Eng., 2001; A312: 248-252 [17] Lavrentiev V.; Abe A.; Yamamoto S.; Naramoto H.; Narumi K.; Formation of promising Co-C nanocpmposites. Sur. Inter. Anal., 2005; 35: 3639

[18] Wang Z. H.; Zhang Z. D.; Choi C. J.; Kim B. k.; Structure and magnetic properties of $\mathrm{Fe}(\mathrm{C})$ and $\mathrm{Co}(\mathrm{C})$ nanocapsules prepared by chemical vapor condensation. J. All. Comp., 2003; 361: 289-293

[19] Cheng J.; Hu P.; Ellis P.; French S.; Kelly G.; Lok M.; Density functional theory study of 
iron and cobalt carbides for Fischer-Tropsch synthesis. J. Phys. Chem. C, 2010; 114: 10851093

[20] Zhang Y.; Li J.; Zhou L.; Xiang S.; A theoretical study on the chemical bonding of 3dtransion-metal carbides. Sol. Stat. Comm., 2002; 121: 411-416

[21] Tzeli D.; Mavridis A.; Electronic structure of cobalt carbide CoC. J. Phys. Chem. A, 2006; 110: 8952-8962

[22] Durgun E.; Senger R. T.; Mehrez M.; Dag S,; Ciraci S.; Nanospintronic properties of carbon-cobalt atomic chains. Europhys. Let., 2006; 73: 642-648

[23] Yong-Hui H.; Su H. Y.; Sun K.; Liu J.; Li W. X.; Structural and electronic properties of cobalt carbide $\mathrm{Co}_{2} \mathrm{C}$ and its surface stability; Density functional theory study. Sur. Sci., 2012; 606: 598-604

[24] Diaz Barriga-Arceo L.; Orozco E.; GaribayFebles V.; Bucio-Galindo L.; Mendoza Leon M.; Castillo-Ocampo P. et. al.; Nanofibre growth from cobalt carbide produced by mechanosynthesis, J. Phys.: Cond. Matt, 2004; 16: S2273-S2277
[25] Partnoi V. K.; Leonov A. V.; Mechanical synthesis of Co-C materials In. Mater., 2012; 48 : $593-600$

[26] Chinnasamy C. N.; Huang J. Y.; Lewis L. H.; Latha B.; Vittoria C.; Harris V. G.; Direct chemical synthesis of high coercivity air-stable SmCo nanoblades. Appl. Phys. Lett., 2008; 93:

032505-032508

[27] Harris V. G.; Chen Y.; Yang A.; Yoon S.; Chen Z.; Geiler A. L. et. al.; High coercivity cobalt carbide nanoparticles processed via polyol reaction: a new permanent magnet materia. Phys. D: Appl. Phys., 2010; 43: 165003-165010 [28] Zamanpour M.; Bennett S. P.; Taheri P.; Chen Y.; Harris V. G.; Magnetic properties and scale-up of nanostructured cobalt carbide permanent magnetic powders. J. Appl. Phys., 2014; 115: 17A7471-17A7474

[29] Kodama D.; Shinoda K.; Sato K.; Sato Y.; Jeyadevan B.; Tohji K.; Synthesis of Fe-Co alloy particles by modified polyol process. IEEE Trans. Magn., 2006; 42: 2796-2798

[30] Zamanpour M.; Chen Y.; Carroll K.; Carpenter E. E.; Lewis L. H.; Harris V. G. et. al.; Large-scale synthesis of high moment FeCo 
nanoparticles using modified polyol synthesis. J. Appl. Phys., 2011; 111: 7-10

[31] Carroll K. J.; Huba Z. H.; Spurgeon S. R.; Qian M.; Khana S. N.; Hudgins D. M.; et. al.; Magnetic properties of Co2C and Co3C nanoparticles and their assemblies, Appl. Phys., 2012; 101: 012409-012414

[32] Kodama D.; Kazo S.; Kimitaka S.; Mann D.; Liu Z.; et. al.: FeCo/graphitic-shell Yoshihiro K.; Joseyphus R. J.; Kenichi M.; et. al. nanocrystals as advanced magnetic-resonanceChemical Synthesis of Sub-micoometer to imaging and near-infrared agents. Nat. Mater., Nanometer size Magnetic FeCo Dice. Adv. 2006; 5: 971-97

Mater., 2006; 18: 3154-3159
[33] Willard M. A.; Kurihara L. K.; Carpenter E. E.; Calvin S.; Harris V. G.; Chemically prepared magnetic nanoparticles. Inter. mater. rev., 2004; 49: $125-170$

[34] Rosen M. J.; Kunjappu J. T; Interfacial Phenomena. John Wiley \& Sons, 2010, 1-98 [35] Seo W. S.; Lee J. H.; Sun X.; Suzuki Y.; $2006 ; 5: 971-97$ 